\title{
Cluster headache with Brugada electrocardiogram pattern: a case report
}

\author{
Yuka Matsuki · Munetaka Hirose • Akira Nakano • \\ Katsuhiko Sarasawa · Toshio Hamada
}

Received: 1 May 2008/Accepted: 4 June 2008/Published online: 19 June 2008

(C) Springer-Verlag 2008

\begin{abstract}
Agents including the effect on sodium channel are restricted in patients with Brugada electrocardiogram (ECG) pattern or Brugada syndrome. On the other hand, many therapeutic agents for cluster headache $(\mathrm{CH})$ block sodium channel. After recommended therapies without sodium channel blocking effect were failed in a 40 -yearold male with $\mathrm{CH}$ whose ECG shows Brugada ECG pattern, clonazepam and codein phosphate, which are exceptional treatments for $\mathrm{CH}$, were effective for severe unilateral orbital pain.
\end{abstract}

Keywords Brugada electrocardiogram pattern .

Clonazepam $\cdot$ Cluster headache $\cdot$ Codein

\section{Introduction}

Cluster headache $(\mathrm{CH})$ is one of the syndromes of trigeminal autonomic cephalalgias, which is characterized by severe unilateral pain localized to orbital, temporal, and midface areas accompanied by typical autonomic symptoms [1]. On the other hand, Brugada syndrome is known for a cause of sudden death due to ventricular fibrillation,

Y. Matsuki · M. Hirose $(\square)$

Department of Anesthesiology and Reanimatology, Faculty of

Medical Sciences, University of Fukui, Eiheijicho, Yoshidagun,

Fukui 910-1193, Japan

e-mail: hirosem@u-fukui.ac.jp

A. Nakano $\cdot$ K. Sarasawa

First Department of Internal Medicine, Faculty of Medical

Sciences, University of Fukui, Fukui, Japan

T. Hamada

Department of Neurology, Fukui Prefectural Hospital,

Fukui, Japan and is characterized by ST-segment elevation in the precordial electrocardiogram (ECG) leads (V1-3) associated with right bundle branch block [2, 3]. The same ECG pattern in patients without clinical symptoms (e.g., syncope, cardiac arrest, family history, etc.) is diagnosed as Brugada ECG pattern [4, 5]. Prevalence of $\mathrm{CH}$ is estimated to be $0.1-0.4 \%$ [1] and that of Brugada ECG pattern is $0.1 \%$ [5]. Therefore, a case of $\mathrm{CH}$ with Brugada ECG pattern is likely very rare.

Agents including the effect of sodium channel block are restricted in patients with Brugada ECG pattern or Brugada syndrome, as these have the potential to induce sudden cardiac arrest. Many treatments for $\mathrm{CH}$, however, include sodium channel blocking effect, such as local anesthetics, lithium, and verapamil $[1,6]$. Therefore, it would be difficult to treat severe headache in a case for $\mathrm{CH}$ patient accompanied with either Brugada syndrome or Brugada ECG pattern, especially if therapies without the effect on sodium channel were failed.

\section{Case report}

A 40-year-old Japanese male, who was diagnosed with Brugada ECG pattern (Fig. 1), was complained of $\mathrm{CH}$. $\mathrm{He}$ had no history of ventricular tachycardia/fibrillation and familial sudden cardiac death.

The characteristics of pain was a severe right orbital pain occurring spontaneously at least one time in a day, lasting for 3 months in a year. This headache associated with tearing eyes and rhinorrhea. Alcohol intake aggravated the attacks of pain. Sumatriptan or oxygen inhalation had been used for symptomatic treatment, and prednisolone and valproic acid had been used as preventive treatment for over 10 years in Fukui Prefectural Hospital. Severity of 


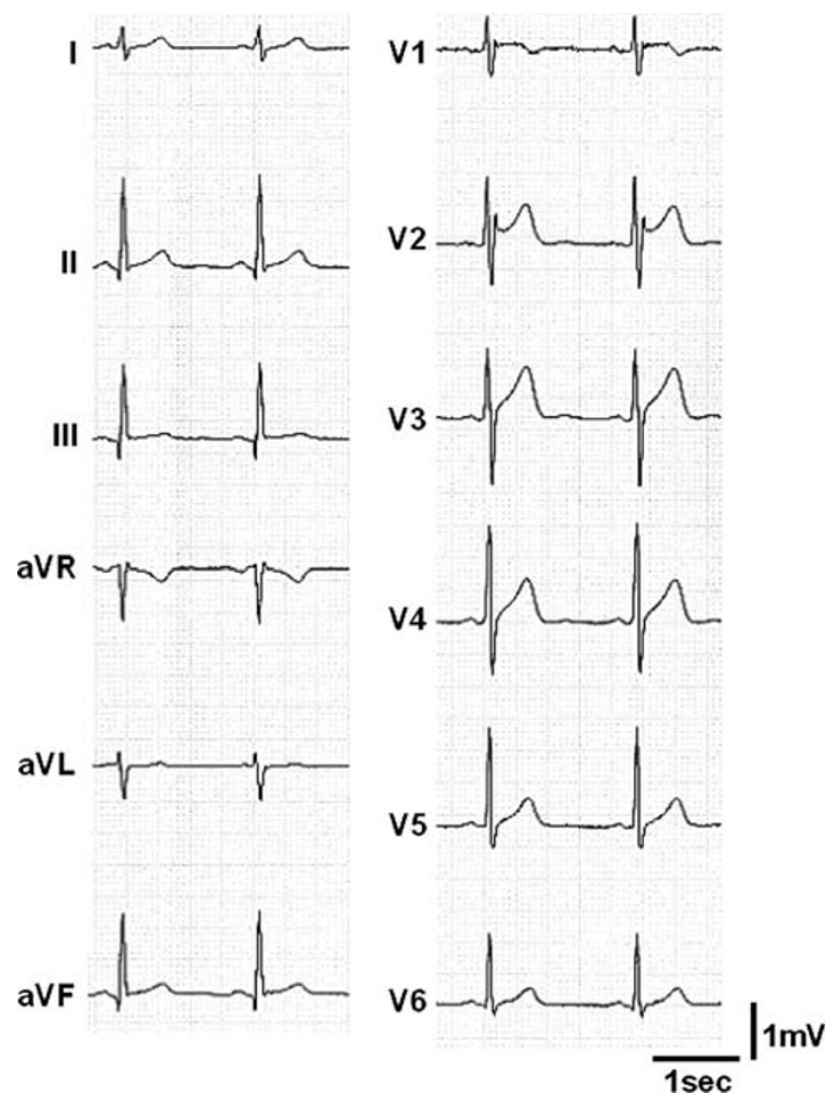

Fig. 1 Saddle-back type ST-segment elevation in lead V2

headache, however, has been aggravated gradually in spite of these treatments, and he was inquired for further treatment in Pain Clinic in University of Fukui, Faculty of Medical Sciences.

Although we prescribed zolmitriptan (2.5 mg p.o.) for attack and lomerizine (10-20 mg/day p.o.) for prevention at first, severity of headache and the number of attacks showed no change after prescription for 2 weeks. Local anesthestics for nasal instillation and nerve blocks, and also verapamil were not selected, because these agents block sodium channel and can induce lethal arrhythmias in a patient with Brugada syndrome or Brugada ECG pattern [7-11]. Then, we selected clonazepam and codein phosphate, which are exceptional treatments for $\mathrm{CH}[6,12-14]$. Clonazepam (2 mg/day p.o.) and codein phosphate (120 mg/day p.o.) suppressed severity of headache dramatically without ECG change. Frequency of attacks, however, showed no change.

\section{Discussion}

The ECG patterns in Brugada syndrome are divided into two types in association with a shape of ST-segment ele- vation; coved- and saddle-back type. Sodium channel gene mutations are associated with Brugada syndrome, and therapeutic use of agents including sodium channel blocking effect is prohibited as it can induce ventricular fibrillation [2, 3]. Individuals with Brugada ECG pattern also have a risk of sudden death [4]. Local anesthetics block sodium channel. Epidural anesthesia with bupivacaine unmasked a subclinical form of Brugada ECG pattern or Brugada syndrome [7, 8]. Thoracic paravertebral block with ropivacaine induced ventricular tachycardia in a patient with Brugada syndrome [9]. Verapamil, a calcium blocker, also blocks sodium channel. Verapamil, used in patient with Brugada syndrome, caused ventricular fibrillation $[10,11]$.

Treatments for $\mathrm{CH}$ is divided into acute therapy aimed at aborting individual attacks and prophylactic therapy aimed at preventing recurrent attacks $[1,6]$. Acute therapy includes inhalation of $100 \%$ oxygen, nasal instillation of lidocain, subcutaneous sumatriptan, and ergot derivatives. As preventive therapy, there are verapamil, lithium, corticosteroids, and nonsurgical and surgical procedures (e.g., greater occipital nerve block, neurostimulation of the occipital nerve) $[1,6]$. Among these recommended therapeutics, nasal instillation of lidocaine, nerve block with local anesthetics, and verapamil cannot be used in patients of $\mathrm{CH}$ accompanying with Brugada syndrome.

Clinical management with regard to the treatment for sudden cardiac death of asymptomatic patients with Brugada ECG pattern has yet to be determined [5]. Not all patients with Brugada ECG pattern are affected by Brugada syndrome. Mediastinal tumor is reported to cause Brugada ECG pattern [15]. Therefore, if careful examination eliminated a risk of sudden cardiac death in patients with both $\mathrm{CH}$ and Brugada ECG pattern, therapeutic agents for $\mathrm{CH}$ including sodium channel blocking effect could be used in these patients.

We treated a patient suffering from $\mathrm{CH}$ associated with Brugada ECG pattern, after the recommended therapeutics without the effect of sodium channel block were failed. As a risk of sudden cardiac death was not completely eliminated in this patient, we decided not to use agents including sodium channel blocking effect. Prescription of clonazepam and codein phosphate decreased the intensity of headache, although frequency of the attack showed no change. Both opioids and anticonvulsants without sodium channel blocking effects, such as clonazepam, gabapentin, and baclofen might be a therapeutic choice for $\mathrm{CH}$ accompanied with Brugada syndrome or Brugada electrocardiogram pattern. Surgical procedures, such as neurostimulation of the occipital nerve, might also be substituted for the treatment in this patient. 


\section{Conflict of interest None.}

\section{References}

1. Balasubramaniam R, Klasser GD (2007) Trigeminal autonomic cephalalgias. Part 1: cluster headache. Med Oral Pathol Oral Radiol Endod 104:345-358

2. Brugada R, Brugada J, Antzelevitch C, Kirsch GE, Potenza D, Towbin JA, Brugada P (2000) Sodium channel blockers identify risk for sudden death in patients with ST-segment elevation and right bundle branch block but structurally normal hearts. Circulation 101:510-515

3. Francis J, Antzelevitch C (2005) Brugada syndrome. Int J Cardiol 101:173-178

4. Brugada J, Brugada R, Brugada P (2003) Determinants of sudden cardiac death in individuals with the electrocardiographic pattern of Brugada syndrome and no previous cardiac arrest. Circulation 108:3092-3096

5. Di Grande A, Tomaselli V, Massarelli L, Amico S, Bellanuova I, Barbera A (2006) Brugada-like electrocardiographic pattern: a challenge for the clinician. Eur J Intern Med 17:3-7

6. Saper JR, Silberstein SD, Gordon CD, Hamel RL, Swidan S (1999) Handbook of headache management, 2nd edn. Lippincott Williams \& Wilkins, Baltimore

7. Phillips N, Priestley M, Denniss R, Uther JB (2003) Brugada-type electrocardiographic pattern induced by epidural bupivacaine. Anesth Analg 97:264-267
8. Vernooy K, Sicouri S, Dumaine R, Hong K, Oliva A, Burashnikov E, Timmermans C, Delhaas T, Crijns HJGM, Antzelevitch C, Rodriguez L-M, Brugada R (2006) Genetic and biophysical basis for bupivacaine-induced ST segment elevation and VT/VF. Anesthesia unmasked Brugada syndrome. Heart Rhythm 3:10741078

9. Fujiwara Y, Shibata Y, Kurokawa S, Satou Y, Komatsu T (2006) Ventricular tachycardia in a patient with Brugada syndrome during general anesthesia combined with thoracic paravertebral block. Anesth Analg 102:1590-1591

10. Fish JM, Antzelevitch C (2004) Role of sodium and calcium channel block in unmasking the Brugada syndrome. Heart Rhythm 1:210-217

11. Chinushi M, Tagawa M, Nakamura Y, Aizawa Y (2006) Shortening of the ventricular fibrillatory intervals after administration of verapamil in a patient with Brugada syndrome and vasospastic angina. J Electrocardiol 39:331-335

12. Caccia MR (1975) Clonazepam in facial neuralgia and cluster headache. Clinical and electrophysiological study. Eur J Neurol 13:560-563

13. Sicuteri F, Raino L, Geppetti P (1983) Substance P and endogenous opioids: how and where they could play a role in cluster headache. Cephalalgia 3(suppl 1):143-145

14. Takebayashi M, Fujikawa T, Kagaya A, Horiguchi J, Yamawaki S (1999) Lithium and clonazepam treatment of two cases with cluster headache. Psychiatry Clin Neurosci 53:535-537

15. Tarin N, Farre J, Rubio JM, Tunon J, Castro-Dorticos J (1999) Brugada-like electrocardiographic pattern in a patient with a mediastinal tumor. Pacing Clin Electrophysiol 22:1264-1266 